\title{
Scapulothoracic muscle strength in individuals with neck pain
}

\author{
Shannon M. Petersen ${ }^{\mathrm{a}, *}$, Nathan A. Domino ${ }^{\mathrm{a}}$ and Chad E. Cook ${ }^{\mathrm{b}}$ \\ ${ }^{a}$ Physical Therapy Program, Des Moines University, Des Moines, IA, USA \\ ${ }^{\mathrm{b}}$ Research Division of Physical Therapy, Duke Medical Center, Durham, NC, USA
}

\begin{abstract}
.
BACKGROUND: People with neck pain often present with weakness in the scapulothoracic muscles. Few studies have examined lower trapezius (LT), middle trapezius (MT) and serratus anterior (SA) muscle strength in individuals with neck pain, nor compared strength to asymptomatic individuals.

OBJECTIVES: The aim of this study was to examine LT, MT and SA muscle strength in individuals with chronic neck pain. METHODS: Descriptive cross sectional design. Twenty two individuals with chronic neck pain and 17 asymptomatic individuals were included. Participants were asked to complete a screening questionnaire, Neck Disability Index, and underwent manual muscle testing for the LT, MT, and SA muscles bilaterally. Data analyses included paired and comparative independent t-tests.

RESULTS: For individuals with neck pain, significant within subject differences in strength between sides for the LT $(P<0.01)$ and MT $(P<0.01)$ were present. In contrast, no within subject difference between sides for the asymptomatic group was found for any muscle. Individuals with neck pain were significantly weaker than asymptomatic individuals for the LT $(p=0.02)$, MT ( $p=0.03)$, and SA $(p=0.01)$ on their side of neck pain, but not on their non-painful side.

CONCLUSIONS: Significant within subject differences were found between sides for the LT and MT while significant between group differences were identified for all three muscles tested.
\end{abstract}

Keywords: Axioscapular, dyskinesis, serratus anterior, scapula, trapezius

\section{Introduction}

Muscle weakness is a finding that has been associated with neck pain. Previous studies have shown impaired cervical flexor muscle performance in subjects with neck pain [1] and exercises directed toward these muscles has been shown beneficial [2]. There is emerging evidence that scapulothoracic muscle weakness may also be associated with neck pain although relatively few studies on this topic exist in the literature $[3,4]$.

Childs [5] published the Neck Pain: Clinical Practice Guidelines Linked to the International Classification

\footnotetext{
*Corresponding author: Shannon M. Petersen, Physical Therapy Program, Des Moines University, 3200 Grand Avenue, Des Moines, IA 50312, USA. Tel.: +1 515271 1688; Fax: +1 515271 7082; E-mail: Shannon.petersen@dmu.edu.
}

of Functioning, Disability, and Health from the Orthopaedic Section of the American Physical Therapy Association (CG). These CGs describe evidence-based physical therapy practice for management of patients with musculoskeletal neck pain. They state that coordination, strength and endurance deficits may be present in the scapulothoracic muscles. Specifically weakness in the lower trapezius (LT), middle trapezius (MT), and serratus anterior (SA) may be present in people who fit into the specific category of neck pain with movement coordination impairments. Coordination, strengthening, and endurance exercises are recommended interventions for individuals fitting into this category in addition to those fitting into the neck pain with mobility deficit category, however there is little evidence indicating impaired muscle function is present in those who fit into the mobility deficit category.

A study by Shahidi [4], examining scapulothoracic muscle strength in people with and without

ISSN 1053-8127/16/\$35.00 (c) 2016 - IOS Press and the authors. All rights reserved

This article is published online with Open Access and distributed under the terms of the Creative Commons Attribution Non-Commercial License (CC BY-NC 4.0). 
chronic neck pain found significantly less strength in the MT and rhomboid muscles of symptomatic individuals compared to asymptomatic individuals. This study however, found no significant difference between groups for the LT. Petersen and Wyatt [3] did find significant weakness in the LT when comparing the ipsilateral side of neck pain versus the contralateral side in individuals with neck pain. This finding was irrespective of side of hand dominance. No studies to date have compared MT strength between painful and nonpainful sides in individuals with neck pain. Additionally, strength of these muscles has not been examined in individuals who fit into the neck pain with mobility deficit classification as defined in the physical therapy CG [5].

Few studies have examined the SA in individuals with neck pain. No studies have tested SA strength using a clinical strength measure; however there is evidence that impaired muscle behavior may be present in this population. Altered SA muscle activation has been found in individuals with neck pain during arm elevation [6]. Sheard [7] also found abnormal firing patterns in the SA of individuals with neck pain. Despite these findings, no investigations have compared SA strength between sides in individuals with neck pain, nor have they compared SA strength in people with and without neck pain.

The purpose of this study was to examine scapulothoracic muscle strength on the side of neck pain compared to the asymptomatic side in individuals with neck pain who fit the $\mathrm{CG}$ neck pain with mobility deficit category. A second purpose was to compare LT, MT, and SA muscle strength in people with and without neck pain.

\section{Methods}

\subsection{Design}

This cross sectional study was designed to describe within-subject and between-subject comparisons.

\subsection{Ethical considerations}

All participants signed a written informed consent form. This study received approval from the University’s Institutional Review Board.

\subsection{Subjects}

Participants in both the neck pian group and the asymptomatic group were recruited from the same university community through flyers posted in common areas. Recruitment for both groups was open to male and female subjects between the ages of 18 and 65 years of age.

Individuals in the neck pain group met criteria based on the Impairment-Based Category of Neck Pain with Mobility Deficit according to CG [5]. Specifically, the inclusion criteria were:

- Unilateral neck pain

- Limited cervical range of motion (ROM)

- Neck pain reproduced at end ranges of active and passive motions

- Restricted cervical and thoracic segmental mobility

- Neck pain or related upper extremity pain reproduced with provocation of the involved spinal segment

- No pain in the scapular or shoulder region.

To be included in the asymptomatic group, participants had to be free of neck and shoulder pain. Individuals were excluded from either group if they had red flags on their medical screening form, if they reported current use of blood thinners, if they had a history of whiplash injury within the previous six weeks, demonstrated evidence of central nervous system involvement, exhibited pathological reflexes, or demonstrated two or more positive neurologic signs consistent with nerve root compression. Individuals were not eligible to participate if they reported previous spinal surgery, workers compensation case or pending litigation related to their neck pain, or had insufficient English language skills to complete the required questionnaires.

\subsection{Procedure}

Screening for inclusion in the study was performed by a physical therapist. Prior to strength assessment, individuals in the neck pain group completed the Neck Disability Index (NDI). Active cervical ROM was measured with a MicroFET 6 dual-site inclinometer. Subjects were asked to move their head and neck into the directions of cervical flexion, extension, rotation to the right and left and lateral flexion to the right and left. They were asked to move as far as possible into each direction. This method has previously been found reliable [8]. Cervical and thoracic segmental mobility was 
examined for mobility and pain provocation. This was done with the patient in a prone position; the examiner provided an oscillatory pressure over each spinal segment. Previous reports have shown this method to be reliable for patients with neck pain [9]. A second researcher, who was blind to the results of the screening examination, tested strength of the LT, MT, and SA. The researcher performing the strength measures was also blind to the participants' group allocation (neck pain or asymptomatic).

\subsection{Strength testing}

A microFET2 digital handheld dynamometer was used to assess strength of the LT, MT, and SA. The order of muscle tested was randomized and the order of extremity tested was randomized. Each muscle was tested 2 times, and the average was recorded. Participants were positioned in prone for testing of the LT and MT while the SA was tested in a seated position. Measurements were recorded in Newtons (N). Handheld dynamometry has been shown to be highly reliable for both inter-rater and intra-rater testing $[10,11]$ and has been determined a valid method for strength assessment $[12,13]$.

Prior to strength testing, participants were given a verbal description of each test procedure. Strength of each muscle was tested in the standard manual muscle test position as described by Kendall [14]. For testing of the LT, participants were positioned prone with a towel roll under their forehead. The extremity being tested was positioned diagonally overhead, in line with the muscle fibers and the scapula was passively positioned in an adducted and depressed position. All participants were able to attain the start position of the test. The examiner provided manual fixation just inferior to the contralateral scapula. Participants were asked to maintain the arm position. The examiner provided pressure with the dynamometer in a downward direction over the distal third of the radial forearm until the participants' maximal effort was overcome (Fig. 1).

For testing of the MT, participants were positioned in prone with their shoulder in 90 degrees of shoulder abduction, with the shoulder in lateral rotation. The scapula was positioned into adduction. The examiner provided manual fixation just inferior to the contralateral scapula to prevent trunk rotation during testing. Participants were asked to maintain their arm position as the examiner applied a downward force with the dynamometer over the distal third of the radial forearm until the participants' maximal effort was overcome (Fig. 2).

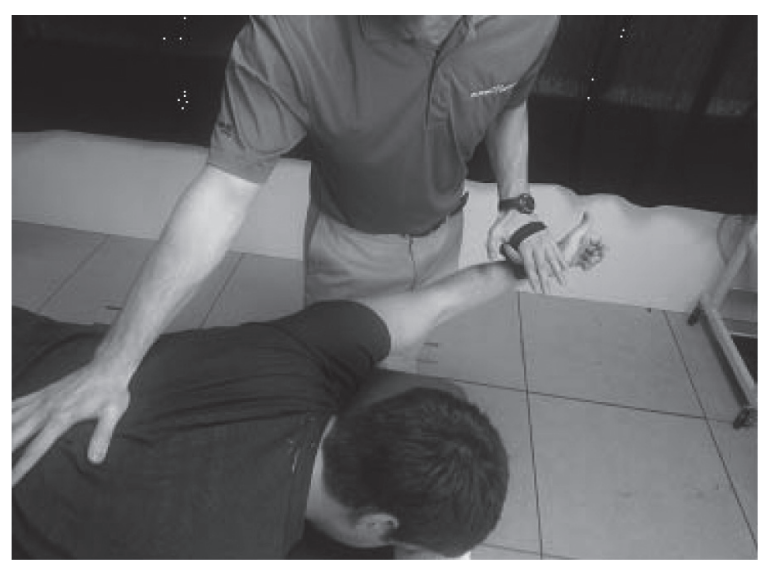

Fig. 1. Lower trapezius muscle test position.

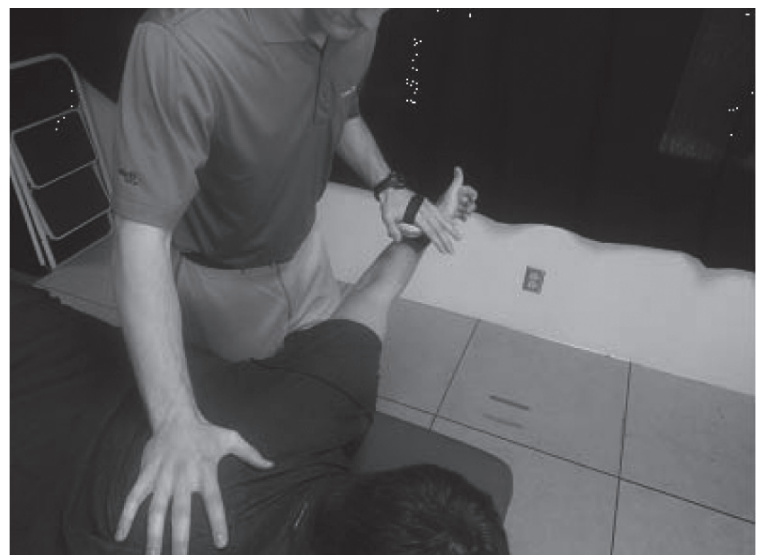

Fig. 2. Middle trapezius muscle test position.

For testing of the SA, participants were seated in a standard chair with their feet flat on the floor and back supported by the back rest. Their arm was positioned with scapular abduction and the shoulder flexed to 125 degrees. Participants were asked to maintain the upper extremity position as the examiner provided a downward force with the dynamometer just proximal the elbow. The strength measurement was collected at the point the break occurred during the test (Fig. 3).

\subsection{Data analysis}

Sample size estimation was performed for the primary purpose of the study. Using a comparative analysis of two dependent groups at a power of $80 \%$, alpha of 0.05 , and effect size of 0.6 , the minimum sample size of 19 was estimated for statistical significance.

Descriptive statistics were used for gender, age, hand dominance, symptom duration, and NDI score. 


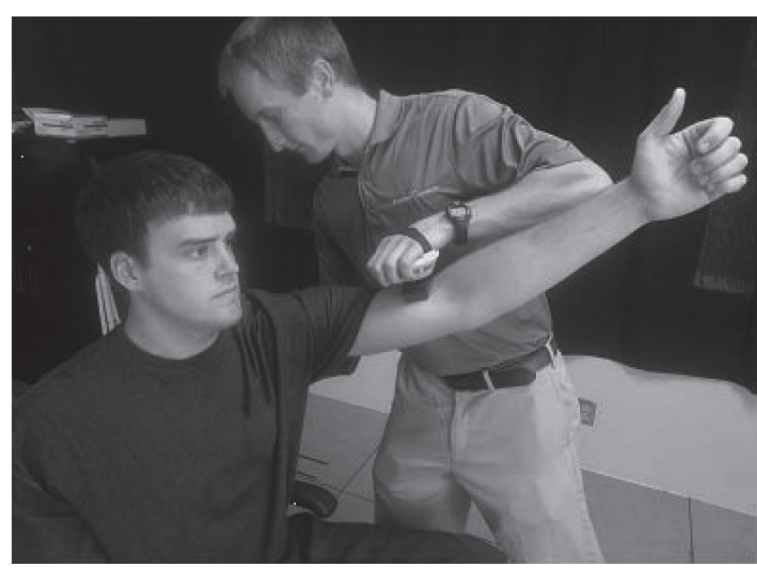

Fig. 3. Serratus anterior muscle test position.

Paired t-tests were used to determine if there was a difference in strength between painful and non-painful sides in individuals with neck pain and to determine if there was a difference in strength between right and left sides for the asymptomatic group. Independent ttests were used to determine if there was a difference in strength between individuals with neck pain and asymptomatic individuals.

Intraclass correlation coefficients (ICC) with $95 \%$ Confidence Intervals (CI) were calculated on a sub-set of subjects to determine between-trial intrarater reliability. This was done on all subjects in the asymptomatic control group for each muscle. Reliability was examined on a total of 34 limbs for the LT, MT, and SA muscles.

\section{Results}

Participants included 22 individuals with neck pain and 17 asymptomatic individuals between the ages of 22 and 63 years. There was no significant difference between groups for age, gender, height, weight, or hand dominance. Demographic information is summarized in Table 1. Intrarater reliability as indicated by the ICCs varied by muscle examined. ICCs (CI) for each muscle were as follows: LT $0.92(0.84-0.96)$, MT 0.94 (0.90-0.96), SA 0.83 (0.66-0.91).

Means for strength of each muscle on the painful side and non-painful side are presented in Tables 2 and 3 for the neck pain group and the strength on right and left sides are presented for the asymptomatic group. In the neck pain group, a significant difference was found between painful and non-painful sides for LT $(P<$ $0.01)$ and MT $(P<0.01)$; although there was no sig- nificant difference between sides for the SA. In the asymptomatic group, there was no within-group difference between sides for any of the muscles (Table 2). Individuals with neck pain were significantly weaker in the limb ipsilateral to the side of neck pain compared to asymptomatic group (Table 3 ). In contrast, there were no significant findings for the LT and MT when the non-painful side in individuals with neck pain was compared to the asymptomatic group. The SA was found to be significantly weaker in the symptomatic group, even on the non-painful side $(P<0.01)$.

\section{Discussion}

Findings of this study suggest significantly lower scapulothoracic muscle strength values in individuals with neck pain compared to those without. Lower strength values were found on the painful side in individuals with neck pain; additionally, those with neck pain had lower strength values compared to the asymptomatic group.

Within-subject differences between sides in LT and MT strength were found in the neck pain group; but these differences did not exist in those without pain. One potential explanation may be due to postural asymmetries in those with neck pain. Scapulothoracic postural impairment has been previously demonstrated during functional tasks in patients with neck pain [15]. Furthermore, correcting poor scapular posture has been shown to normalize scapulothoracic muscle activity during a functional task in patients with neck pian [16]. Since we did not specifically examine scapulothoracic postural deviations or muscle length in this study, we do not know whether asymmetries observed were related to muscle imbalances or scapular position [17].

Another potential reason for impaired strength in those with neck pain is that the pain itself may prevent individuals from exerting their full effort upon strength testing. It is possible that subjects did not put forth their maximal effort during muscle testing for fear of potential increased pain, therefore resulting in lower strength measures.

The finding that the LT was weaker on the side of neck pain is consistent with that of Petersen [3]. The finding that MT weakness was present compared to the asymptomatic group is consistent with the findings of Shahidi [4], however in contrast to their finding for the LT which did not show differences between groups. One potential reason for the difference observed is that 
Table 1

Demographic information

\begin{tabular}{llll}
\hline Variables & Neck pain group & Asymptomatic group & P value \\
\hline Age (years) Mean (SD) & $31.8(12.9)$ & $32.2(11.6)$ & 0.92 \\
Height (in cm) & $169.93(7.44)$ & $173.91(11.79)$ & 0.10 \\
Weight (in kg) & $70.43(14.42)$ & $78.47(26.99)$ & 0.24 \\
Hand Dominance & $20=$ Right handed & $16=$ Right handed & $1=$ Left handed \\
Side of Symptoms & $2=$ Left handed & N/A & \\
& $12=$ Right & & \\
Gender & $10=$ Left & $10=$ Male & \\
& $19=$ Male & $7=$ Female & \\
Mean (SD) Symptom Duration (months) & $3=$ Female & N/A & N/A \\
NDI Score Mean (SD) & $43.27(62.71)$ & N/A & N/A \\
\hline
\end{tabular}

Table 2

Within-group strength

\begin{tabular}{|c|c|c|c|c|c|c|}
\hline Side (group) & $\begin{array}{l}\text { Painful side } \\
\text { (NP) }\end{array}$ & $\begin{array}{l}\text { Non-painful side } \\
\text { (NP) }\end{array}$ & $\begin{array}{l}\text { Within-group } \\
\text { (NP) P-Value }\end{array}$ & $\begin{array}{l}\text { Right side } \\
\text { (AG) }\end{array}$ & $\begin{array}{l}\text { Left side } \\
\text { (AG) }\end{array}$ & $\begin{array}{l}\text { Within-group } \\
\text { (AG) P-Value }\end{array}$ \\
\hline Lower trapezius & $18.9(6.5)$ & $21.5(9.4)$ & $<0.01^{*}$ & $24.4(8.5)$ & $23.9(8.8)$ & 0.86 \\
\hline Middle trapezius & $22.1(10.2)$ & $24.5(9.7)$ & $<0.01^{*}$ & $28.8(11.5)$ & $28.9(11.3)$ & 0.98 \\
\hline Serratus anterior & 36.7 (18.7) & $38.0(19.9)$ & 0.10 & $55.8(27.2)$ & $54.7(19.7)$ & 0.83 \\
\hline
\end{tabular}

Mean (Standard Deviation) of strength in Newtons for each muscle. For subjects with neck pain (NP), the painful and non-painful sides are presented. For subjects in the asymptomatic group (AG), right and left are presented. P-Values are presented for within-group findings between sides for both the NP group and the AG group.

Table 3

Between-group strength

\begin{tabular}{|c|c|c|c|c|c|c|c|c|c|}
\hline Side (group) & $\begin{array}{c}\text { Painful } \\
\text { side (NP) }\end{array}$ & $\begin{array}{c}\text { Right } \\
\text { side }(A G)\end{array}$ & $\begin{array}{c}\text { Between-group } \\
\text { (NP/AG) } \\
\text { P-Values }\end{array}$ & $\begin{array}{c}\text { Non-painful } \\
\text { side (NP) }\end{array}$ & $\begin{array}{l}\text { Left side } \\
\text { (AG) }\end{array}$ & $\begin{array}{c}\text { Between-group } \\
\text { (NP/AG) } \\
\text { P-values }\end{array}$ & $\begin{array}{c}\text { Average both } \\
\text { sides (NP) }\end{array}$ & $\begin{array}{c}\text { Average both } \\
\text { sides (AG) }\end{array}$ & $\begin{array}{l}\text { Between-group } \\
\text { (NP/AG) } \\
\text { P-values }\end{array}$ \\
\hline Lower trapezius & $18.9(6.5)$ & $24.4(8.5)$ & $0.02^{*}$ & $21.5(9.4)$ & $23.9(8.8)$ & 0.21 & $20.2(8.1)$ & $24.0(8.5)$ & $0.02 *$ \\
\hline Middle trapezius & $22.1(10.2)$ & $28.8(11.5)$ & $0.03^{*}$ & $24.5(9.7)$ & 28.9 (11.3) & 0.10 & $23.3(9.9)$ & $28.9(11.2)$ & $0.01^{*}$ \\
\hline Serratus anterior & 36.7 (18.7) & $55.8(27.2)$ & $0.01^{*}$ & $38.0(19.9)$ & 54.7 (19.7) & $<0.01^{*}$ & $37.4(19.1)$ & $55.2(23.4)$ & $<0.01^{*}$ \\
\hline
\end{tabular}

Mean (Standard Deviation) of strength in Newtons for each muscle. P-Values presented are for between-group findings comparing subjects with neck pain (NP) to asymptomatic group (AG) subjects. For subjects in the AG group, the right side was defined as the painful side and left side was defined as the non-painful side.

Shahidi [4] performed the LT and MT tests by pressure application above the participants' elbow while the current study applied pressure to the forearm as described by Petersen [3] and by Kendall [14]. Test pressure application over the forearm results in a longer lever arm which may potentially be more difficult to maintain in a painful population. Neither study examined muscle performance during a functional task so it is uncertain if this difference would translate to function. Findings for the MT however were consistent between studies in that both found weakness in participants with neck pain compared to those without [4].

Findings for SA strength differed from those of the LT and MT. Although significantly weaker on the painful side in individuals with neck pain compared to the asymptomatic group, there was no withinsubject difference between painful and non-painful sides. Standard deviations for the SA were much higher than for the other muscles which may have impacted the results observed. Perhaps subject or examiner characteristics may have influenced the wide variation in scores observed for the SA. For example subject height may have positioned them differently in the chair resulting in a differing force provided by the examiner. Our small sample size may have also contributed to the wide standard deviation. Previous studies have demonstrated impaired SA activation during functional tasks in people with neck pain, but there are no previous studies of specific strength testing of this muscle so our results cannot be compared [6,7].

Another potential reason for differences observed is that this study examined individuals who fit the specific CG criteria of neck pain with mobility deficit while the previous studies did not attempt to distinguish between subjects with differing types of neck pain [5]. International Statistical Classification of Diseases and Related 
Health Problems (ICD) and the International Classification of Functioning, Disability, and Health (ICF) indicate scapulothoracic muscle impairment may be used to diagnose patients with sprain and strain of cervical spine and neck pain with movement coordination impairments respectively [5]. The current study however shows that individuals who have neck pain with mobility deficit can also present with limited strength in this muscle group.

\subsection{Limitations}

This study has some limitations that may impact application of results. The sample size was small, and was a sample of convenience. Because this was a cross sectional study it shows strength only at one point in time, and does take into account changes in strength relative to chronicity of symptoms. There was no statistically significant difference in gender between groups in our study, but it possible that the difference may be clinically significant. There were more males in the neck pain group, and this group showed lower strength values on the painful side; it is possible that gender difference between groups may have contributed to the differences observed however one may expect males to demonstrate greater strength than females and not lower strength values as we found in our study. It is possible that a greater between-group difference may have been observed if the groups were more closely matched for gender. The age of our subjects is a potential limiting factor. Despite having no difference in age between groups, our subjects were relatively young, so generalizing our results to an older population may not be applicable. Additionally, intervention was not a component of this study, so no conclusions can be drawn regarding management of patients who have scapulothoracic muscle weakness associated with their neck pain.

\section{Conclusion}

Muscle weakness in the LT, MT, and SA muscles is present in individuals with neck pian. LT and MT are weaker on the side of neck pain compared to the contralateral side. Strength of these muscles should be examined in patients who present with neck pain. Further investigation is warranted to determine the best management strategies and to determine if improvements in strength correspond to improvements in neck pain and function.

\subsection{Implications for physiotherapy practice}

Clinicians should examine scapulothoracic muscle strength in patients who present with neck pain.

\section{Conflict of interest}

The authors have no conflict of interest to report.

\section{References}

[1] Falla D, Jull G, Hodges P. Patients with neck pain demonstrate reduced electromyographic activity of the deep cervical flexor muscles during performance of the craniocervical flexion test. Spine. 2004; 29(19): 2108-2114.

[2] Jull GA, Falla DL, Vicenzino B, Hodges PW. The effect of therapeutic exercise on activation of the deep cervical flexor muscles in people with chronic neck pain. Man Ther. 2009; 14(6): 696-701.

[3] Petersen SM, Wyatt SN. Lower trapezius muscle strength in individuals with unilateral neck pain. J Orthop Sports Phys Ther. 2011; 41(4): 260-265.

[4] Shahidi B, Johnson C, Curran-Everett D, Maluf K. Reliability and group differences in quantitative cervicothoracic measures among individuals with and without chronic neck pain. BMC Musculoskelet Disord. 2012; 13: 215.

[5] Childs JD, Cleland JA, Elliott JM, Teyhen DS, Wainner RS, Whitman JM, et al. Neck pain: clinical practice guidelines linked to the International Classification of Functioning, Disability, and Health from the Orthopaedic Section of the American Physical Therapy Association Journal of Orthopedic and Sports Physical Therapy. J Orthop Sports Phys Ther. 2008; 38(9): A1-A34.

[6] Helgadottir H, Kristjansson E, Einarsson E, Karduna A, Jonsson $\mathrm{JrH}$. Altered activity of the serratus anterior during unilateral arm elevation in patients with cervical disorders. J Electromyogr Kinesiol. 2011; 21(6): 947-953.

[7] Sheard B, Elliot J, Cagnie B, O’Leary S. Evaluating serratus anterior muscle function in neck pain using muscle functional magnetic resonance imaging. J Manipulative Physiol Ther. 2012; 35(8): 629-635.

[8] Cleland JA, Childs JD, Fritz JM, Whitman JM. Interrater reliability of the history and physical examination in patients with mechanical neck pain. Arch Phys Med Rehabil. 2006; 87: 1388-1395.

[9] Jull G, Zito G, Trott P, Potter H, Shirley D. Inter-examiner reliability to detect painful upper cervical joint dysfunction. Aust J Physiother. 1997; 43(2): 125-129.

[10] Berg HE, Berggren G, Tesch PA. Dynamic neck strength training effect on pain and function. Arch Phys Med and Rehabil. 1994; 75(6): 661-665.

[11] Bohannon RW, Andrews AW. Interrater reliability of handheld dynamometry. Phys Ther. 1987; 67(6): 931-933.

[12] May LA, Burnham RS, Steadward RD. Assessment of isokinetic and hand-held dynamometer measures of shoulder rotator strength among individuals with spinal cord injury. Arch Phys Med and Rehabil. 1997; 78(3): 251-255. 
[13] Michener LA, Boardman ND, Pidcoe PE, Frith AM. Scapular muscle tests in subjects with shoulder pain and functional loss: reliability and construct validity. Phys Ther. 2005; 85(11): 1128-1138.

[14] Kendall FP, McCreary EK, Provance PG, Rodgers MM, Romani WA. Muscles: Testing and Function With Posture and Pain. 5th ed. Baltimore: Lippincott, Williams, \& Wilkins; 2005, pp. 329-333.
[15] Szeto GPY, Straker L, Raine S. A field comparison of neck and shoulder postures in symptomatic and asymptomatic office workers. Appl Ergon. 2002; 33(1): 75-84.

[16] Wegner S, Jull G, O'Leary S, Johnston V. The effect of a scapular postural correction strategy on trapezius activity in patients with neck pain. Man Ther. 2010; 15(6): 562-566.

[17] Zakharova-Luneva E, Jull G, Johnston V, O'Leary S. Altered trapezius muscle behavior in individuals with neck pain and clinical signs of scapular dysfunction. J Manipulative Physiol Ther. 2012; 35(5): 346-353 